\title{
ÜBER LIMITIERUNGSVERFAHREN, DIE VON EINEM STIELTJES-INTEGRAL ABHÄNGEN.
}

\author{
VoN
}

G. G. LORENTZ

in Frankfurt am Main.

Einführung.

In dieser Arbeit untersuchen wir Limitierungsverfahren der Form

$$
\sigma(x)=\int_{0}^{+\infty} a(x, t) d s(t) .
$$

Existiert für die Funktion $\sigma(x)$, die für alle $x \geqq 0$ sinnvoll sein soll, der endliche Grenzwert $\lim _{x \rightarrow \infty} \sigma(x)=\sigma$, so ist $\sigma$ der verallgemeinerte Grenzwert, der durch das Verfahren (I) der Funktion $s(t)$ (für $t \rightarrow \infty$ ) zugeordnet wird. Das Verfahren (I) heisst konvergenztreu, falls es jeder konvergenten Funktion $s(t) \rightarrow s$ aus einer gewissen Funktionenklasse einen Grenzwert $\lim \sigma(x)=\sigma$ zuordnet, und permanent, wenn dabei stets $\sigma=s$ ist.

Damit das Integral (I) existiert, muss zunächst das Stieltjes-Integral

$$
\int_{0}^{c} a(x, t) d s(t)
$$

für jedes endlicbe $c>0$ vorhanden sein (das Integral ( $I$ ) ist dann der Grenzwert dieses letzteren für $c \rightarrow \infty$ ). Es gibt nun zwei Wege, die Existenz der Integrale (2) zu sichern, indem man eine der beiden folgenden Annahmen macht:

I. $a(x, t)$ ist für jedes feste $x \geqq 0$ eine stetige Funktion von $t$ und $s(t)$ ist in jedem Intervall $(o, c)$ von beschränkter Schwankung.

2. Es ist umgekehrt $s(t)$ stetig und $a(x, t)$ ist von beschränkter Schwankung in jedem Intervall $(o, c)$.

Zunächst betrachten wir kurz den zweiten Fall. Es ist leicht zu beweisen, dass das Integral $(\mathrm{I})$ genau dann für jede stetige Funktion $s(t)$ mit endlichem 
$\lim _{t \rightarrow \infty} s(t)$ existiert, wenn $a(x, t)$ bei jedem $x \geqq 0$ im ganzen Intervall $0 \leqq t<+\infty$ von beschränkter Schwankung ist. In diesem Fall verhält sich das Integral (1) im Wesentlichen wie ein Stieltjes-Integral für ein endliches Intervall und bat alle wichtigsten Eigenschaften eines solchen. Insbesondere kann man eine partielle Integration ausführen und die Transformation ( 1 ) dadurch in die Form

$$
\sigma(x)=\int_{0}^{\infty} s(t) d_{t} b(x, t)
$$

bringen mit einer passend zu wählenden Funktion $b(x, t)$, die in $0 \leqq t<+\infty$ von beschränkter Schwankung sein wird. Sätze über die Konvergenztreue und die Permanenz für das Verfahren (I) im Falle 2. ergeben sich somit automatisch aus den entsprechenden Sätzen für das duale Verfahren (3). (Wir bezeichnen als dual zueinander Verfahren, welche durch eine formale partielle Integration in $(0,+\infty)$ in einander übergehen.) Diese letzteren Sätze können aus einem Satz von F. RIEsz ${ }^{1}$ abgeleitet werden, der die notwendigen und hinreichenden Bedingungen für die Konvergenz einer Folge

$$
\lim _{n \rightarrow \infty} \int_{0}^{+\infty} s(t) d b_{n}(t)=\int_{0}^{\infty} s(t) d b(t)
$$

angibt, wobei $b_{n}(t), n=\mathrm{I}, 2, \ldots$ und $b(t)$ feste Funktionen von beschränkter Schwankung in $(0,+\infty)$ und $s(t)$ eine beliebige stetige Funktion bedeutet, für die $\lim s(t)$ vorhanden ist. Wir wollen uns daher im Falle 2. mit diesen kurzen Bemerkungen begnügen und ihn dadurch als erledigt ansehen.

Anders liegen die Dinge im Fall I. Hier lässt sich das Limitierungsverfahren (I) jm Allgemeinen nicht in die Form (3) bringen, weil das erste Integral nur als ein uneigentliches Stieltjes-Integral existiert und die Umformung durch partielle Integration in ( 1 ) nicht mehr möglich ist.

Man kommt indessen ganz natürlich auf die Limitierungsverfahren (I) mit den Voraussetzungen I., wenn man eine Transformation für die Funktion $s(t)$ sucht, die die Transformation

${ }^{1}$ F. Rresz, Comptes Rendus, Paris 149 (1909), 974-977.

2 Dieser Satz von F. Riesz ist ein Spezialfall eines allgemeineren Satzes über konvergente Folgen von linearen Funktionalen $F_{n}(s)$ in einem Banachschen Raum $S=\{s\}:$ die Folge $F_{n}(s)$ konvergiert genau dann in $S$ gegen ein lineares Funktional $F(s)$, wenn sie 1 ) auf einer Menge der 8 gegen $F(s)$ konvergiert, deren lineare Hülle überall dicht in $S$ liegt und wenn 2) die Normen $\left\|F_{n}\right\|$ beschränkt sind. Vgl. etwa S. BANACH, Théorie des opérations, Warszawa 1932, S. $79-80$. 


$$
\sigma_{n}=\sum_{v=0}^{\infty} a_{n v} u_{v}
$$

der Folge $s_{n}=\sum_{v=0}^{n} u_{v}$ als Spezialfall enthält. Setzt man nämlich

$$
s(t)=\left\{\begin{array}{l}
\text { o für } t=0 \\
s_{v} \text { für } v<t \leqq v+\mathrm{I}, y=0, \mathrm{I}, \ldots
\end{array}\right.
$$

so hat diese Treppenfunktion an den Stellen $t=\nu$ die Sprünge $u_{v}$, während sie sonst streckenweise konstant ist. Daher ist für sie

$$
\sigma(x)=\int_{0}^{\infty} a(x, t) d s(t)=\sum_{v=0}^{\infty} a(x, \nu) u_{v},
$$

was der Transformation (4) entspricht mit $x$ statt $n$ als neue Veränderliche. Unter den Voraussetzungen 2. aber führt das Ansetzen der Funktion (5) in (I) nicht zum Ziel, da die Existenz des Integrals (I) nur für stetige Funktionen $s(t)$ gesichert ist.

Die Hauptaufgabe dieser Arbeit besteht darin, die Bedingungen für die Permanenz und die Konvergenztreue des Verfahrens ( $\mathrm{I}$ ) unter den Bedingungen $\mathrm{I}$. zu finden $\left(\S_{1}\right)$ und dann die Zusammenhänge zwischen den dualen Transformationen (I) und (3) zu untersuchen $(\$ 2)$. Im letzten $\$ 3$ bringen wir endlich einige Anwendungen und Spezialfälle.

\section{§ I. Konvergenztreue und permanente Verfahren.}

Es sei $\mathfrak{S}_{\mathfrak{v}}$ die Gesamtheit der Funktionen $s(t)$, die für $t \geqq 0$ definiert und in jedem endlichen Intervall $(o, c)$ ron beschränkter Schwankung sind. Ferner nehmen wir an, dass in jedem Punkt $t>0$ der Wert $s(t)$ zwischen den Grenzwerten $s(t \pm 0)$ in diesem Punkte liegt, der Einfachheit halber sogar dass er gleich dem arithmetischen Mittel dieser Grenzwerte ist. Diese Eigenschaft kann immer durch eine Änderung der Funktion $s(t)$ in höchstens abzählbare vielen Punkten erzwungen werden; der Wert der Integrale (I) bleibt dabei ungeändert, während die totale Schwankung der Funktion $s(t)$ in jedem endlichen Intervall ihren kleinsten Wert annimmt.

Auf die Frage, unter welchen Bedingungen das Integral ( 1 ) für ein festes $x$ existiert, antwortet der

17-61491112 Acta mathematica. 79 
Satz 1. Damit das Integral

$$
\int_{0}^{\infty} a(t) d s(t)
$$

fïr alle bei $t \rightarrow \infty$ konvergenten Funttionen $s(t)$ aus $\mathcal{S}_{v}$ existiert, ist notwendig und hinreichend, dass a) a(t) stetig ist und b) eine Zahl $c>0$ existiert, so dass

$$
\underset{(c, \infty)}{\operatorname{Var}} a(t)<+\infty
$$

ist. Es ist dann $a(t)$ in $(0, \infty)$ beschränkt und $\lim _{t \rightarrow \infty} a(t)=a$ vorhanden.

Dem Beweis schicken wir den folgenden bekannten Hilfssatz voraus:

Hilfssatz 1. Es sei $a(t)$ für $\alpha \leqq t \leqq \beta$ stetig und es sei $\operatorname{Var} a(t)>b \geqq 0$. Dann gibt es zu jeder Zahl $l>0$ eine in $\langle\alpha, \beta\rangle$ erklärte Funktion von beschränkter Schwankung, für die

$$
s(\alpha)=s(\beta)=0, \quad|s(t)| \leqq l, \quad \text { und } \int_{a}^{\beta} a(t) d s(t)>b l
$$

ist.

Beweis. Wir können Punkte $\alpha<\alpha_{1}<\beta_{1}<\alpha_{2}<\cdots<\beta_{r}<\beta$ derart angeben, dass

$$
\sum_{i=1}^{r}\left|a\left(\beta_{i}\right)-a\left(\alpha_{i}\right)\right|>b
$$

ist. Für jedes $i$ setzen wir $s(t)=l_{i}=l \cdot \operatorname{sign~}\left[a\left(\alpha_{i}\right)-a\left(\beta_{i}\right)\right]$ im offenen Intervall $\left(\alpha_{i}, \beta_{i}\right)$ und $=0$ in den übrigen Punkten von $\langle\alpha, \beta\rangle$, so dass $s(t)$ in dem Punkte $\alpha_{i}$ den Sprung $l_{i}$, in dem Punkte $\beta_{i}$ den Sprung $-l_{i}$ macht. Diese Funktion ist in $\langle\alpha, \beta\rangle$ von beschränkter Schwankung, genügt den beiden ersten Forderungen (7) und für sie ist auch

$$
\int_{\alpha}^{\beta} a(t) d s(t)=\sum_{i=1}^{r} l_{i}\left[a\left(\alpha_{i}\right)-a\left(\beta_{i}\right)\right]=l \sum\left|a\left(\beta_{i}\right)-a\left(\alpha_{i}\right)\right|>b l .
$$

Beweis des Satzes. Die Bedingung a) ist notwendig. Denn ist $a(t)$ etwa im Punkt $t_{0}$ unstetig, so existiert das Integral (6) sicher nicht, wenn $s(t)$ eine Funktion aus $\mathfrak{S}_{v}$ ist, die in diesem Punkt $t_{0}$ einen Sprung $s\left(t_{0}+0\right)-s\left(t_{0}-0\right)$ macht, der $\neq 0$ ist.

Auch b) ist notwendig. Denn ist diese Bedingung nicht erfüllt, so gibt es eine Folge von Intervallen $\left\langle\alpha_{k}, \beta_{k}\right\rangle$ ohne gemeinsame Punkte derart, dass $\alpha_{k} \rightarrow \infty$ strebt für $k \rightarrow \infty$ und dass $\underset{\left\langle\alpha_{k}, \beta_{k}\right\rangle}{\operatorname{Var}} a(t)>k^{2}$ ist. Nach dem Hilfssatz gibt es dann 
in $\left\langle\alpha_{k}, \beta_{k}\right\rangle$ eine Funktion $s(t)$ von beschränkter Schwankung unit $|s(t)|<\frac{1}{k}$, $s\left(\alpha_{k}\right)=s\left(\beta_{k}\right)=0, \int_{\alpha_{k}}^{\beta_{k}} a(t) d s(t)>k$. Wird $s(t)$ ausserhalb der Intervalle $\left\langle\alpha_{k}, \beta_{k}\right\rangle$ gleich Null gesetzt, so erhält man eine gegen o konvergierende Funktion aus $\mathfrak{S}_{v}$, für die das Integral (6) nicht existiert.

Die Bedingungen sind auch hinreichend. Sie sichern zunächst die Existenz des Integrals $\int_{0}^{c} a(t) d s(t)$ bei jedem $c>0$. Da ferner die Veränderung von $s(t)$ um eine additive Konstante auf die Existenz des Integrals (6) ohne Einfluss ist, so darf $\lim s(t)=0$ angenommen werden und diese Existenz folgt aus

$$
\int_{\alpha}^{\beta} a(t) d s(t)=a(\beta) s(\beta)-a(\alpha) s(\alpha)-\int_{\alpha}^{\beta} s(t) d a(t)
$$

durch eine triviale Abschätzung für grosse $\alpha$ und $\beta$.

Satz 2. Die Integraltransformation (I) ist dann und nur dann konvergenztreu, wenn bei jedem $x \geqq 0$ die Funktion a $(x, t)$ die im Satz I formulierten Eigenschaften a) und b) hat und wenn die folgenden Bedingungen erfüllt sind:

$\left(N_{1}\right)$ Es existieren endliche positive Zahlen $c, M$ und $x_{0}$ derart, dass

$$
\underset{\langle c, \infty)}{\operatorname{Var}} a(x, t) \leqq M \text { ist fiir } x \geqq x_{0} .
$$

$\left(N_{\varrho}\right)$ Es ist $a(x, t)$ gleichmässig beschränkt für $x \geqq x_{0}$, etwa

$$
|a(x, t)| \leqq K \text { fïr } x \geqq x_{0}, t \geqq 0 .^{3}
$$

(L) Es ist $\lim _{x \rightarrow \infty} a(x, t)=a^{*}(t)$ für jedes $t \geqq 0$ vorhanden.

Sind diese Bedingungen erfillt und ist $a^{*}(t)$ ausserdem stetig, so wird der Grenzwert $\lim _{x \rightarrow \infty} \sigma(x)=\sigma$ durch die Formel

$$
\sigma=\int_{0}^{\infty} a^{*}(t) d s(t)
$$

geliefert. fordern.

s Wegen $\left(N_{1}\right)$ würde es hier genügen, die gleichmässige Beschränktheit in $0 \leqq t \leqq c$ zn 
Beweis. I. Die Bedingungen sind notwendig. Für die Bedingung (L) erkennt man das sofort mit Hilfe der Funktion

$$
s(t)= \begin{cases}\text { o für } t \leqq t_{0} \\ 1 \text { für } t>t_{0} .\end{cases}
$$

Denn für sie ist $\sigma(x)=a\left(x, t_{0}\right)$. Wir dürfen also weiterhin $(L)$ als erfüllt ansehen.

Die Notwendigkeit von $\left(N_{1}\right)$ beweisen wir dadurch, dass wir, wofern sie nicht erfüllt ist, eine Funktion $s(t) \in \mathfrak{S}_{v}$ angeben, für die das Integral (I) nicht existiert. Ist nämlich $\left(N_{1}\right)$ nicht erfüllt, so gibt es zu jedem $c>0$ und $M>0$ beliebig grosse $x$, für die

$$
\underset{c \leqq t=\infty}{\operatorname{Var}} a(x, t)>M
$$

ausfällt. Wird also $c_{1}>0$ beliebig gewählt, so gibt es ein $x_{1}>0$ so dass $\underset{\left(c_{1}, \infty\right)}{\operatorname{Var}} a\left(x_{1}, t\right)>\mathrm{I}$ ist und folglich ein $d_{1}>c_{1}$, so dass auch noch $\underset{\left(c_{1}, d_{1}\right)}{\operatorname{Var}} a\left(x_{1}, t\right)>\mathrm{I}^{2}$ ist. Nach Bedingung b) von Satz I kann dieses $d_{1}$ zugleich so gross gewählt werden, dass

$$
\underset{\left(d_{1}, \infty\right)}{\operatorname{Var}} a\left(x_{1}, t\right)<\mathrm{I}
$$

ist. Nun setzen wir die Funktion $s(t)=0$ in $\left\langle 0, c_{1}\right\rangle$ und in $d_{1}$ und gemäss Hilfssatz I in $\left(c_{1}, d_{1}\right)$ so, dass dort $|s(t)| \leqq I$ und

$$
\int_{c_{1}}^{d_{1}} a\left(x_{1}, t\right) d s(t)>1^{2}
$$

ausfällt. Sind nun schon die Intervalle $\left\langle c_{1}, d_{1}\right\rangle, \ldots,\left\langle c_{k-1}, d_{k-1}\right\rangle$ mit $c_{1}<d_{1}<$ $<c_{2}<\cdots<d_{k-1}$ und die Zahlen $x_{1}<x_{2}<\cdots<x_{k-1}$ bestimmt und ist die Funktion $s(t)$ schon in $\left\langle 0, d_{k-1}\right\rangle$ erklärt, so wird folgendermassen fortgefahren:

Es seien die Zahlen $\bar{x}_{k}$ und $g_{k}$ so gross gewählt, dass für $x \geqq \bar{x}_{k}$

$$
\left|\int_{0}^{d_{k-1}} a(x, t) d s(t)\right| \leqq g_{k}
$$

ausfällt. Solche Zahlen $\hat{x}_{k}$ und $g_{k}$ gibt es, da das abgeschätzte Integral bei $x \rightarrow \infty$ einen Grenzwert besitzt. ${ }^{4}$ Weiter wird $c_{k}>d_{k-1}$ beliebig und $x_{k}>\bar{x}_{k}$ so gewählt, dass $\underset{\left(c_{k}, \infty\right)}{\operatorname{Var}} a\left(x_{k}, t\right)>k g_{k}+k^{2}$ ist, und schliesslich $d_{k}>c_{k}$ so gross dass schon

4 Denn es ist gleich dem Integral (1), wenn man dort $8(t)$ für $0 \leqq t \leqq d_{k-1}$ gleich der obigen Funktion $s(t)$, für $t>d_{k-1}$ aber gleich 0 wählt. 


$$
\underset{\left(c_{k}, d_{k}\right)}{\operatorname{Var}} a\left(x_{k}, t\right)>k g_{k}+k^{2}, \text { aber } \underset{\left\langle d_{k}, \infty\right)}{\operatorname{Var}} a\left(x_{k}, t\right)<\mathrm{I}
$$

ist. Die Funktion $s(t)$ setzen wir dann in $\left(d_{k-1}, c_{k}\right)$ gleich Null und erklären sie in $\left\langle c_{k}, d_{k}\right\rangle$ nach dem Hilfssatz 2 so, dass

$$
s\left(c_{k}\right)=s\left(d_{k}\right)=0,|s(t)| \leqq \frac{1}{k} \text { und } \int_{c_{k}}^{d_{k}} a\left(x_{k}, t\right) d s(t)>\frac{\mathrm{I}}{k}\left(k g_{k}+k^{2}\right)
$$

ist. Die so erklärte Funktion $s(t)$ gehört zu $\Im_{v}$ und es strebt $s(t) \rightarrow 0$; es ist aber

$$
\left|\sigma\left(x_{k}\right)\right|=\left|\int_{u}^{\infty} a\left(x_{k}, t\right) d s(t)\right| \geqq\left|\int_{c_{k}}^{d_{k}}\right|-\left|\int_{0}^{c_{k}}\right|-\left|\int_{d_{k}}^{\infty}\right| \geqq g_{k}+k-g_{k}-\mathrm{I}=k-\mathrm{I},
$$

wie sich aus den Abschätzungen in (12) und (10) und, für das dritte Integral, durch eine partielle Integration von $\int_{d_{k}}^{a}$ für $d \rightarrow \infty$ ergibt. Denn $a\left(x_{k}, t\right)$ ist nach (I I) für $t \geqq d_{k}$ beschränkt und strebt für $t \rightarrow \infty$ einem Grenzwert zu.

Schliesslich ist auch die Bedingung $\left(N_{2}\right)$ notwendig. Man erkennt dies am einfachsten mit Hilfe des in der Fussnote 2) angeführten Satzes. Ersetzt man nämlich die Werte einer Funktion $s(t) \in \mathfrak{S}_{v}$ durch Null für $t \geqq e$, so erhält man, dass für eine beliebige Funktion $s(t)$, die von beschränkter Schwankung in $\langle\mathrm{o}, c\rangle$ ist,

$$
\lim _{x \rightarrow \infty} \int_{0}^{c} a(x, t) d s(t)
$$

vorhanden sein muss. Diese Funktionen $s(t)$ bilden einen Banachschen Raum $V$,

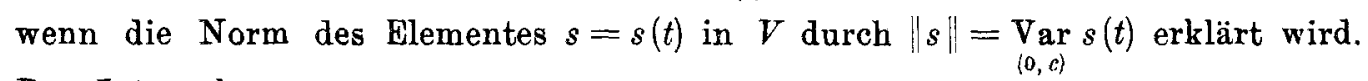
Das Integral

$$
F(s)=\int_{0}^{c} a(t) d s(t)
$$

stellt dann bei stetigem $a(t)$ ein lineares Funstional im Raume $V$ dar, und die Norm von $F(s)$ ist gleich Max $|a(t)| .^{5}$ Aus der Konvergenz der Funktionale

$$
\int_{0}^{c} a(x, t) d s(t)
$$

bei $x \rightarrow \infty$ folgt also in der Tat die Bedingung $\left(N_{\mathbf{g}}\right)$.

s Vgl. z. B. BaNAch, a. a. O., S. 59 und $189-190$. 
II. Die Bedingungen sind hinveichend. Zunächst ist das Integral (14) für jedes $c>0$ und jedes $s(t) \in \Im_{v}$ bei $x \rightarrow \infty$ konvergent. Denn ist $s(t)$ monoton nicht abnehmend, $t(s)$ die $\mathrm{zu} s(t)$ inverse Funktion, so ist das Integral (14) gleich dem Lebesgueschen Integral

$$
\int_{s(0)}^{s(c)} a(x, t(s)) d s
$$

Da hier der Integrand bei festem $s$ nach $(L)$ für $x \rightarrow \infty$ konvergiert und für alle in Betracht kommenden $x$ und $s$ dem Betrage nach unter $K$ liegt, so konvergiert das Integral bei $x \rightarrow \infty$ nach dem Lebesgueschen Konvergenzsatz gegen

$$
\int_{s(0)}^{s(e)} a^{*}(t(s)) d s\left(=\int_{0}^{c} a^{*}(t) d s(t), \text { falls } a^{*}(t) \text { stetig ist }\right)
$$

Dasselbe gilt, wenn $s(t)$ monoton nicht wachsend ist, und deshalb auch für jede Funktion von beschränkter Schwankung $s(t)$.

Um die Existenz des Grenzwertes $\lim _{x \rightarrow \infty} \sigma(x)$ nachzuweisen, genügt es nun zu zeigen, dass das Integral $\int_{c}^{d} a(x, t) d s(t)$, also auch das Integral $\int_{c}^{\infty} a(x, t) d s(t)$ für hinreichend grosse $c$ und $d$ beliebig klein wird und zwar gleichmässig für alle hinreichend grossen $x$. Da nun die Änderung von $s(t)$ um eine additive Konstante in diesem Integral dessen Wert nicht beeinflusst, so dürfen wir bej diesem Nachweis $\lim s(t)=0$ annehmen und dieser ergibt sich sofort aus

$$
\int_{c}^{d} a(x, t) d s(t)=a(x, d) s(d)-a(x, c) s(c)-\int_{c}^{d} s(t) d a(x, t)
$$

durch einfachste Abschätzung.

Da die Differenz der Integrale in (14) und (15) bei stetigem $a^{*}(t)$ für hinreichend grosse $x$ beliebig klein ist und für hinreichend grosse $c$ sich das erste von $\sigma(x)$, das zweite von dem Integral (8) beliebig wenig unterscheidet, so folgt schliesslich auch die Richtigkeit der Formel (8). Bei dieser hängt $\sigma$ natürlich nicht von dem Wert lim $s(t)=s \mathrm{ab}$, da, wie schon hervorgehoben, ein Stieltjes-Integral durch eine Änderung von $s(t)$ um eine additive Konstante nicht beeinflusst wird.

Aus diesem Grunde ist es nicht möglich, ohne weiteres von der Permanenz der Transformation (I) zu sprechen. Die Lage ändert sich jedoch, wenn man annimmt, dass $s(t)$ etwa durch

$$
s(0)=0
$$

normiert ist, Dann gilt der 
Über Limitierungsverfahren, die von einem Stieltjes-Integral abhängen. 263

Satz 3. Bei der Normierung (16) von $s(t)$ ist die Integraltransformation (1) genau dann permanent, wenn ausser den Bedingungen a) und b) von Satz 1 und den Bedingungen $\left(N_{1}\right)$ und $\left(N_{2}\right)$ von Satz 2 noch

$\left(L_{1}\right)$

$$
\lim _{x \rightarrow \infty} a(x, t)=\mathrm{I} \text { fiir jedes } t \geqq 0
$$

erfiillt ist.

Beweis. Dass $\left(L_{1}\right)$ notwendig ist, ergibt sich wieder mit Hilfe der Funktion (9). Dass es hinreichend ist in Verbindung mit den übrigen Bedingungen, folgt aus der Formel (8), da in diesem Falle

$$
\sigma=\int_{0}^{\infty} d s(t)=\lim _{t \rightarrow \infty} s(t)-s(0)=s
$$

ist.

\section{$\S$ 2. Zusammenhang zwischen den dualen Limitierungsverfahren.}

Für ein konvergenztreues Verfahren

$$
\sigma(x)=\int_{0}^{\infty} a(x, t) d s(t)
$$

existiert nach Satz 2, da $\left(N_{1}\right)$ erfüllt sein muss, der Grenzwert

$$
\lim _{t \rightarrow \infty} a(x, t)=\alpha(x) \text {. }
$$

Über den Wert von $\alpha(x)$ lässt sich folgendes sagen. Ist $\lim _{x \rightarrow \infty} \sigma(x)$ wenigstens fïr eine beschränkte, bei $t \rightarrow+\infty$ divergente Funktion $s(t)$ vorhanden, so ist

$$
\lim _{t \rightarrow \infty} a(x, t)=0, \quad x \geqq 0 \text {. }
$$

Denn beide Integrale in

$$
\int_{0}^{e} a(x, t) d s(t)=a(x, c) s(c)-a(x, 0) s(0)-\int_{0}^{e} s(t) d_{t} a(x, t)
$$

haben für dieses $s(t)$ bei $c \rightarrow \infty$ einen endlichen Grenzwert (das zweite Integral wegen der Bedingung $\left(N_{1}\right)$ und der Beschränktheit der Funktion $s(t)$ ). Daher hat auch $a(x, c) s(c)$ bei $c \rightarrow \infty$ einen endlichen Grenzwert, und da $a(x, c) \rightarrow \alpha(c)$ strebt, muss (ry) erfüllt sein.

Wir wollen wieder $s(t) \in \mathscr{S}_{v}$ in (I) durch die Festsetzung (I6) normieren. Ist noch (I7) erfüllt, so geht aus (I) - zunïchst ganz formal - durch eine partielle Integration 


$$
\sigma(x)=-\int_{0}^{\infty} s(t) d_{t} a(x, t)
$$

hervor, d. h. ein Verfahren vom Typus (3), das wir wieder als dual zu (I) bezeichnen.

Wir wollen jetzt genauer den Zusammenhang zwischen (I) und (I 8) verfolgen. Zunächst beweisen wir einen Hilfssatz über die Umkehrung eines StieltjesIntegrals.

Hilfssatz 2. Es sei $a(t)$ stetig und positiv im Intervall $\langle 0, c\rangle, s(t)$ in ihm von beschränkter Schwankung und

$$
\varrho(y)=\int_{0}^{y} a(t) d s(t)
$$

Dann ist auch $\varrho(y)$ von beschränkter Schwankung und

$$
s(\beta)-s(\alpha)=\int_{\alpha}^{\beta} \frac{d \varrho(t)}{a(t)}, \quad(0 \leqq \alpha<\beta \leqq c) .
$$

Beweis. Es genügt eine monoton nicht abnehmende Funktion $s(t)$ zu betrachten. Dann ist auch $\varrho(y)$ monoton nicht abnehmend, die Existenz der rechten Seite von (20) also gesichert. Dieses Integral ist der Grenzwert der Summen

$$
S=\sum_{i=1}^{n} \frac{\mathrm{I}}{a\left(\boldsymbol{\tau}_{i}\right)}\left[\varrho\left(t_{i}\right)-\varrho\left(t_{i-1}\right)\right],
$$

wenn $\alpha=t_{0}<t_{1}<\cdots<t_{n}=\beta$ eine Zerlegung des Intervalls $\langle\alpha, \beta\rangle$ bedeutet, die $\tau_{i}$ irgendwie zwischen $t_{i-1}$ und $t_{i}$ gewählt sind unc die grösste der Längen der Intervalle $\left\langle t_{i-1}, t_{i}\right\rangle$ gegen Null strebt. Nun ist nach dem Mittelwertsatz

$$
\varrho\left(t_{i}\right)-\varrho\left(t_{i-1}\right)=\int_{t_{i-1}}^{t_{i}} a(t) d s(t)=a\left(\boldsymbol{x}_{i}^{\prime}\right)\left[s\left(t_{i}\right)-s\left(t_{i-1}\right)\right] .
$$

Wird in der Summe $S$ speziell $\tau_{i}=\boldsymbol{i}_{i}^{\prime}$ gewählt, so wird

$$
S=\sum_{i=1}^{n}\left[s\left(t_{i}\right)-s\left(t_{i-1}\right)\right]=s(\beta)-s(\alpha)
$$

und daraus ergibt sich die Behauptung. ${ }^{6}$

- Allgemeiner liefert derselbe Beweis: Es ist unter den angegebenen Bedingungen für eine stetige Funktion $b(t)$

$$
\int_{u}^{\beta} b(t) d s(t)=\int_{\alpha}^{\beta} \frac{b(t)}{a(t)} d \rho(t)
$$


Über Limitierungsverfahren, die von einem Stieltjes-Integral abhängen. $\quad 265$

Satz 4. Es sei $a(t)>0$ und stetig für $0 \leqq t<+\infty$, ferner $\lim _{t \rightarrow \infty} a(t)=0$ und schliesslich

$$
\operatorname{Var}_{0 \leqq t \leqq c} \frac{\mathrm{I}}{a(t)} \leqq \frac{M}{a(c)}
$$

mit einem festen $M \geqq 0$ fïr alle $c>0$. (Diese Bedingung ist insbesondere erfiullt, wenn a $(t)$ monoton gegen o abnimmt.) Existiert dann für ein $s(t) \in \Im_{v}$, das durch (16) normiert ist, das Integral $\int_{0}^{\infty} a(t) d s(t)$, so existiert auch das Integral $\int_{0}^{\infty} s(t) d a(t)$ und es gilt

$$
\int_{0}^{\infty} a(t) d s(t)=-\int_{0}^{\infty} s(t) d a(t) .
$$

Beweis. Es ist

$$
\int_{0}^{x} s(t) d a(t)=a(x) s(x)-\int_{0}^{x} a(t) d s(t)
$$

Wird $\varrho(y)$ durch (I9) erklärt, so ist nach dem Hilfssatz 2

$$
s(x)=s(x)-s(0)=\int_{0}^{x} \frac{d \underline{\varrho}(t)}{a(t)} .
$$

Um den Satz zu beweisen, genügt es also zu zeigen, dass für jede Funktion $\varrho(t) \in \widetilde{S}_{v}$, die für $t \rightarrow \infty$ konvergiert, der Ausdruck

$$
a(x) s(x)=a(x) \int_{0}^{x} \frac{d \varrho(t)}{a(t)}
$$

für $x \rightarrow \infty$ den Grenzwert o hat, oder also dass die Transformation

$$
\int_{0}^{\infty} b(x, t) d \varrho(t) \text { mit } b(x, t)= \begin{cases}\frac{a(x)}{a(t)} & \text { in } 0 \leqq t \leqq x \\ 0 & \text { für } t>x\end{cases}
$$

die Bedingungen des Satzes 2 erfüllt und $\lim b(x, t)=0$ ist für jedes $t \geqq 0$. Das ist aber offenbar der Fall. 
Satz 5. Es seien

$$
\sigma(x)=\int_{0}^{\infty} s(t) d a(x, t)
$$

und

$$
\bar{\sigma}(x)=-\int_{0}^{\infty} a(x, t) d s(t)
$$

zwei duale Limitierungsverfahren, die für die durch (16) normierten $s(t) \in \mathfrak{S}_{v}$ betrachtet werden. Es sei $a(x, t)$ für alle $x \geqq 0$ und $t \geqq 0$ positiv, es sei $\lim _{t \rightarrow \infty} a(x, t)=0$ fiir jedes $x \geqq 0$ und

$$
\operatorname{Var}_{0 \leqq t \leqq c} \frac{\mathrm{I}}{a(x, t)} \leqq \frac{M(x)}{a(x, c)}
$$

mit einem von $c$ freien $M(x)$ oder es sei $a(x, t)$ für jedes $x \geqq 0$ monoton gegen o abnehmend (und dann ist die Beschränkung $a(x, t)>0$ unwesentlich, es darf auch $=0$ sein). Dann ist das Verfahren (23) in dem Verfahren (24) enthalten ${ }^{7}$, es ist sogar. für alle $x \geqq 0$

$$
\sigma(x)=\bar{\sigma}(x) .
$$

Der Beweis folgt unmittelbar aus dem vorangehenden Satz, da der Fall einer monoton abnehmenden Funktion $a(x, t)$, die für hinreichend grosse $t$ gleich o wird, trivial ist.

In der entgegengesetzten Richtung gilt der

Satz 6. Fïr jedes $x \geqq 0$ sei $a(x, t)$ in $(0, \infty)$ von beschränkter Schwankung und es sei fiur $t \geqq 1$

$$
|a(x, t)| \leqq M(x)|a(x, t)-a(x, t-\mathrm{I})| .
$$

Dann ist das Verfahren (24) in dem Verfahren (23) enthalten, wenn man dabei nur solche Funktionen $s(t) \in \mathfrak{S}_{k}$ berïcksichtigt, für die ausser (16) noch die Bedingung erfuillt ist, dass für ein geeignetes $K$

$$
\left|s(t)-s\left(t^{\prime}\right)\right| \leqq K \text { ist für alle } t, t^{\prime} \text { mit }\left|t-t^{\prime}\right|<\mathrm{I} .
$$

Es ist dann sogar wieder $\sigma(x)=\bar{\sigma}(x)$.

Beweis. Wegen

$$
\int_{0}^{e} s(t) d a(x, t)=a(x, c) s(c)-\int_{0}^{e} a(x, t) d s(t)
$$

${ }^{7}$ D. h. jede nach dem ersten Verfahren limitierbare Funktion aus $\mathfrak{G}_{v}$ ist auch nach dem zweiten Verfahren limitierbar und zwar zu demselben Grenzwert. 
Über Limitierungsverfahren, die von einem Stieltjes-Integral abhängen. $\quad 267$ und der Existenz des Integrals in (23) genügt es zu zeigen, dass $a(x, c) s(c) \rightarrow 0$ strebt für jedes $x \geqq 0$ bei $e \rightarrow \infty$. Es ist aber

$$
\begin{aligned}
|a(x, c) s(c)| & \leqq M(x)|a(x, c)-a(x, c-1)||s(c)| \\
& =M(x)\left|\int_{c-1}^{e} s(c) d_{t} a(x, t)\right| \\
& \leqq M(x)\left\{\left|\int_{c-1}^{c} s(t) d a(x, t)\right|+\left|\int_{c-1}^{c}[s(c)-s(t)] d a(x, t)\right|\right\}
\end{aligned}
$$

und hier strebt das erste Integral gegen o, weil das Integral (23) existiert, und das zweite, weil es $\leqq K \operatorname{Var}_{c-1 \leqq t \leqq c} a(x, t)$ ist.

\section{§ 3. Anwendungen und Beispiele.}

Wir behandeln zunächst das Verfahren der bewichteten Mittel $P$

$$
\sigma^{p}(x)=\sigma(x)=\frac{I}{P(x)} \int_{0}^{x} p(t) s(t) d t, \quad P(x)=\int_{0}^{x} p(t) d t,
$$

wobei $p(t)$ - das Gewicht des Verfahrens - für $t \geqq 0$ positiv und stetig und die Funktion $s(t)$ in jedem endlichen Intervall $L$-integrierbar sein soll. Das Verfahren $P$ ist bekanntlich genau dann permanent, wenn $\lim P(x)=\infty$ ist.

Satz 7. Es seien $(P)$ und $(Q)$ zwei solche Verfahren mit den Gewichten $p(t)$ und $q(t)$. Dann ist $P$ in $Q$ enthalten, falls die Funktion

$$
q(t) / p(t), \quad 0 \leqq t<+\infty
$$

monoton nicht wachsend und das Verfahren $Q$ permanent ist.

Beweis. Wir drücken

$$
\sigma^{q}(x)=\frac{\mathrm{I}}{Q(x)} \int_{0}^{x} q(t) s(t) d t=\frac{\mathrm{I}}{Q(x)} \int_{0}^{1} q s d t+\frac{\mathrm{I}}{Q(x)} \int_{1}^{x} q s d t
$$

durch $\sigma(x)=\sigma^{p}(x)$ aus. Da $q(t) / p(t)$ stetig und $p(t) s(t) L$-integrierbar ist, darf das letzte Integral als ein Stieltjes-Integral geschrieben werden. Man erhält so 


$$
\begin{aligned}
\sigma^{q}(x) & =0(\mathrm{I})+\frac{\mathrm{I}}{Q(x)} \int_{1}^{x} \frac{q(t)}{p(t)} d(P(t) \sigma(t)) \\
& =0(\mathrm{I})+\frac{\mathrm{I}}{Q(x)} \int_{0}^{x} q(t) \sigma(t) d t+\frac{\mathrm{I}}{Q(x)} \int_{1}^{x} \frac{q(t)}{p(t)} P(t) d \sigma(t) .
\end{aligned}
$$

Hier stellt das zweite Glied rechts eine permanente Transformation von $\sigma(t)$ dar und konvergiert gegen $\lim _{x \rightarrow \infty} \sigma(x)=s$. Es ist also nur zu zeigen, dass das dritte Glied $\rightarrow$ o strebt; $\sigma(t)$ ist dabei von beschränkter Schwankung im Intervall $(o, x)$ (ja sogar absolut stetig) und für $t \rightarrow \infty$ konvergent. Wir beweisen den Satz indem wir dieses Glied in der Form der Transformation (I) schreiben mit

$$
a(x, t)=\left\{\begin{array}{cll}
\frac{P(t) q(t)}{p(t) Q(x)} & \text { für } & \mathrm{I} \leqq t \leqq x \\
\mathrm{o} & \text { für } t>x
\end{array}\right.
$$

und für diese Transformation die Bedingungen des Satzes 2 nachprüfen. Es ist hier $a^{*}(t)=0$ stetig und also nur noch die Bedingung

$$
\operatorname{Var}_{1 \leqq t \leqq x} \frac{q(t) P(t)}{p(t)} \leqq M Q(x), \quad x \geqq 0
$$

nachzuprüfen. Für ein beliebiges $x \geqq 0$ wählen wir eine Zerlegung $\mathrm{I}=t_{0}<t_{1}<\cdots$ $\cdots<t_{n}=x$ des Intervalls $(\mathrm{I}, x)$, die so dicht sein soll, dass $\left|p(\tau) / p\left(\tau^{\prime}\right)\right|<\mathrm{I}+\varepsilon$, $\left|q(\tau) / q\left(\tau^{\prime}\right)\right|<\mathrm{I}+\varepsilon$ ausfällt, sobald $\tau$ und $\tau^{\prime}$ demselben Teilintervall der Zerlegung angehören. Es sollen $\tau_{v}, \tau_{v}^{\prime}$ durch

$$
\begin{aligned}
& P\left(t_{v}\right)-P\left(t_{v-1}\right)=p\left(\tau_{v}\right)\left(t_{v}-t_{v-1}\right), \\
& Q\left(t_{v}\right)-Q\left(t_{v-1}\right)=q\left(\tau_{v}^{\prime}\right)\left(t_{v}-t_{v-1}\right)
\end{aligned}
$$

definiert sein. Dann ist die Zerlegungssumme für die Schwankung der Funktion $q P / p$ gleich

$$
\begin{aligned}
& \sum_{v=1}^{n}\left|\frac{q\left(t_{v}\right) P\left(t_{v}\right)}{p\left(t_{v}\right)}-\frac{q\left(t_{v-1}\right) P\left(t_{v-1}\right)}{p\left(t_{v-1}\right)}\right| \\
\leqq & \sum_{v=1}^{n} \frac{p\left(\tau_{v}\right) q\left(t_{v}\right)\left(t_{v}-t_{v-1}\right)}{p\left(t_{v}\right)}+\sum_{v=1}^{n} P\left(t_{v-1}\right)\left|\frac{q\left(t_{v}\right)}{p\left(t_{v}\right)}-\frac{q\left(t_{v-1}\right)}{p\left(t_{v-1}\right)}\right| .
\end{aligned}
$$

Die erste Summe rechts ist

$$
\begin{aligned}
& \leqq(1+\varepsilon) \sum_{v=1}^{n} q\left(t_{\nu}\right)\left(t_{v}-t_{\nu-1}\right) \leqq(1+\varepsilon)^{2} \sum_{\nu=1}^{n} q\left(i_{v}^{\prime}\right)\left(t_{v}-t_{v-1}\right) \\
& =(1+\varepsilon)^{2} Q(x) .
\end{aligned}
$$


Die zweite Summe ist (mit $\left.t_{-1}=1\right)$ :

$$
\begin{aligned}
& =\sum_{v=1}^{n} P\left(t_{v-1}\right)\left[\frac{q\left(t_{v-1}\right)}{p\left(t_{v-1}\right)}-\frac{q\left(t_{v}\right)}{p\left(t_{v}\right)}\right] \\
& =P\left(t_{0}\right) \frac{q\left(t_{0}\right)}{p\left(t_{0}\right)}+\sum_{v=0}^{n-1} \frac{q\left(t_{v}\right)}{p\left(t_{v}\right)}\left[P\left(t_{v}\right)-P\left(t_{v-1}\right)\right] \\
& \leqq(1+\varepsilon)^{2} Q(x) .
\end{aligned}
$$

Also gilt $(27)$ mit $M=2(1+\varepsilon)^{9}$.

Folgerungen: 1. Ist $p(t)$ monoton nicht abnehmend, so ist $P$ in dem Verfahren $C_{1}$ der arithmetischen Mittel enthalten: $P<C_{1}$.

2. Ist $p(t)$ monoton nicht wachsend, so gilt $C_{1}<P$. Ganz speziell: das Verfahren der logarithmischen Mittel

enthält $C_{1}$.

$$
\sigma(x)=\frac{\mathrm{I}}{\log x} \int_{1}^{x} \frac{s(t)}{t} d t
$$

Als ein Beispiel der Anwendung der Sätze von $\S 2$ betrachten wir die Reihentransformation

$$
\sigma_{n}=\sum_{v=0}^{\infty} a_{n v} u_{v}
$$

die aus der Transformation ( $I$ ) entsteht, wenn dort die Funktion $s(t)$ die Treppenfunktion (9) ist, $x$ durch die ganzzahlige Veränderliche $n$ ersetzt und $a(n, v)=a_{n v}$ gesetzt wird. Die Funktion $a(n, x)$ denken wir uns für jedes $n$ linear zwischen diesen Werten interpoliert. Die duale Transformation hat hier gemäss dem Formelpaar (23), (24) die Form

$$
\sigma_{n}=\sum_{v=0}^{\infty} b_{n v} \varepsilon_{v}, \quad \quad b_{n v}=a_{n v}-a_{n, v+1}
$$

Aus Satz 5 ergibt sich:

Satz 8. Das Verfahren $B$ ist in dem Verfahren $A$ enthalten, falls $a_{n v}>0$ ist, $a_{n v} \rightarrow 0$ strebt fiir $\nu \rightarrow \infty, n=0, \mathrm{I}, 2, \ldots$ und falls

$$
\sum_{\rho=0}^{v}\left|\frac{1}{a_{n \varrho}}-\frac{1}{a_{n, \rho+1}}\right| \leqq \frac{M_{n}}{a_{n v}}
$$

gilt mit einem $v$-freien $M_{n}$ (insbesondere also wenn $a_{n}$, monoton gegen 0 strebt). 
Dem Satz 6 entspricht hier

Satz 9. Das Verfahren $A$ ist in $B$ enthalten, falls fiir jedes $n=0,1,2, \ldots$ die Reihe $\sum_{\rho=v}^{\infty} b_{n \varphi}=a_{n v}$ absolut konvergiert und fiur alle $v$ gilt

$$
\left|\sum_{\rho=v}^{\infty} b_{n \rho}\right| \leqq M_{n}\left|b_{n v}\right|
$$

Die unangenehme Bedingung (26) des Satzes 6 konnte hier weggelassen werden, weil man im Beweise jenes Satzes im vorliegenden Fall einer Treppenfunktion (9) mit ganzzahligen $c$ auskommt, also die Bedingung (26) nur für $t, t^{\prime}$ braucht, die einem ganzzahligen Intervall $(c, e+1)$ angehören; unter diesen Voraussetzungen ist. (26) trivialerweise von selbst erfüllt.

Aus Satz 8 und 9 folgt:

Satz 10. Die Verfahren $A$ und $B$ sind äquivalent, falls es für jedes $n$ eine Zahl o $<q_{n}<\mathrm{I}$ gibt mit

$$
\mathrm{o} \leqq a_{n, v+1} \leqq q_{n} a_{n v}, \quad n, \nu=0,1,2, \ldots
$$

Entsprechendes gilt natürlich auch, wenn $n$ durch einen stetigen Parameter ersetzt wird. Durch Anwendung dieses Kriteriums erhält man insbesondere leicht:

Das Abelsche

$$
\sigma(r)=\sum_{v=0}^{\infty} r^{v}(\mathrm{I}-r) s_{v}, \quad(\mathrm{o}<r<\mathrm{I}, r \rightarrow \mathrm{I})
$$

und das Borelsche Verfahren

$$
\bar{\sigma}(x)=\sum_{v=0}^{\infty} e^{-x} \frac{x^{v}}{v !} s_{v}, \quad(x \rightarrow+\infty),
$$

die hier die Form $B$ haben, sind den entsprechenden dualen Verfahren $A$ äquivalent.

Zum Schluss wollen wir zeigen, dass im Allgemeinen, wenn keinerlei zusätzliche Voraussetzungen ausser der Permanenz von $A$ oder $B$ gemacht werden, weder $A<B$ noch $B<A$ zu gelten braucht. Genauer zeigen wir durch Gegenbeispiele, dass die Bedingungen der Sätze 8 und 9 nicht ausreichen, um die Äquivalenz der dualen Verfahren zu sichern.

1. Es gibt ein permanentes Verfahren $B$, das die Eigenschaft (29) hat, das aber das duale Verfahren $A\left(\right.$ mit $\left.a_{n v}=\sum_{\rho=v}^{\infty} b_{n \varrho}\right)$ nicht enthält. 
Wir wählen

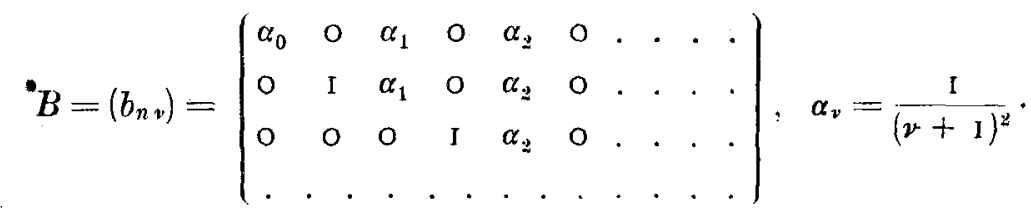

(29) ist offenbar erfüllt. Für die Folge $\left\{s_{\nu}\right\}$ mit $s_{2 \nu}=(-1)^{\nu}(\nu+1)^{3 / 2}, s_{2 v+1}=0$ $(\boldsymbol{v}=0, \mathbf{I}, \ldots)$ strebt die zugehörige $B$-Transformierte

$$
\sigma_{n}=\sum_{\nu=n}^{\infty} \alpha_{\nu} s_{2 \nu}=\sum_{\nu=n}^{\infty}(-\mathrm{I})^{v} \frac{\mathrm{I}}{\sqrt{\nu+\mathrm{I}}} \rightarrow 0 \text { für } n \rightarrow \infty
$$

Dagegen ist für jedes $n=0, I, \ldots$ die Reihe $\Sigma a_{n v} s_{v}$ divergent, denn es ist bei jedem $n$ für hinreichend grosse $v$

$$
a_{n, 2 \nu}=\sum_{\rho=v}^{\infty} \frac{\mathrm{I}}{(\varrho+\mathrm{I})^{2}}
$$

also $=O\left(\frac{1}{v}\right)$, so dass $a_{n, 2 v} s_{2 v} \rightarrow \infty$ strebt für $\nu \rightarrow \infty$.

2. Es gibt ein permanentes Verfahren $A$, das das duale Verfahren $B$ (mit $\left.b_{n v}=a_{n v}-a_{n, v+1}\right)$ nicht enthält, obwohl für $B$ die Bedingung (28) erfiullt ist.

Es sei

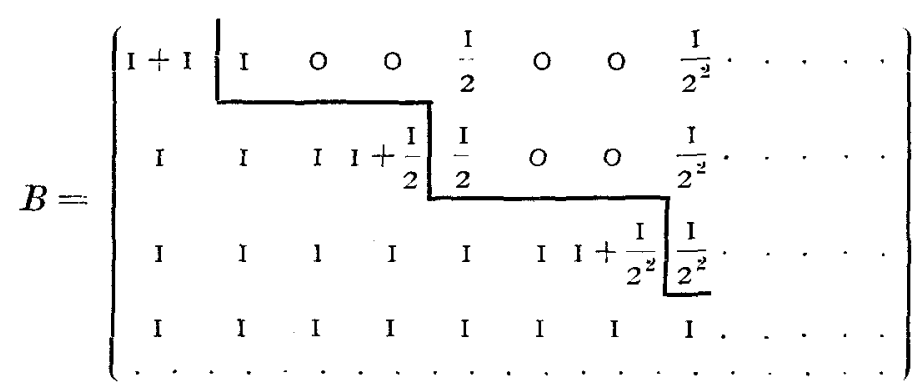

Offenbar gibt es Reihen $\Sigma u_{v}$ mit den Eigenschaften

und

$$
u_{3 v-2}=0, \quad \frac{1}{4^{v}} u_{3 v} \rightarrow+\infty
$$

$$
u_{0}+u_{1}+\cdots+u_{3 v-2}+u_{3 v-1}+\left(I+\frac{1}{2^{v}}\right) u_{3 \nu}=0
$$


272

G. G. Lorentz.

Eine solche Reihe ist $A$-summierbar zum Wert o. $\sigma_{n}$ in $(B)$ dagegen hat für sie keinen Sinn, weil für jedes $n$ und $\nu \geqq 3 n+\mathrm{I}$

$$
b_{n, 3 v+1} s_{3 v+1}=\frac{\mathrm{I}}{2^{v}}\left(-\frac{\mathrm{I}}{2^{v}} u_{3 v}\right)=-\frac{\mathrm{I}}{4^{v}} u_{3 v}
$$

ist, also $b_{n \nu} s_{\nu}$ bei $\nu \rightarrow \infty$ unbeschränkt bleibt. 